\title{
Decay of Rabi oscillations by dipolar-coupled dynamical spin environments
}

\author{
V. V. Dobrovitski, ${ }^{1}$ A. E. Feiguin,${ }^{2,3}$ R. Hanson, ${ }^{4}$ and D. D. Awschalom ${ }^{5}$ \\ ${ }^{1}$ Ames Laboratory, Iowa State University, Ames IA 50011, USA \\ ${ }^{2}$ Condensed Matter Theory Center, Department of Physics, \\ University of Maryland, College Park, MD 20742 \\ ${ }^{3}$ Microsoft Station Q, University of California, Santa Barbara, CA 93106. \\ ${ }^{4}$ Kavli Institute of Nanoscience Delft, Delft University of Technology, \\ P.O. Box 5046, 2600 GA Delft, The Netherlands \\ ${ }^{5}$ Center for Spintronics and Quantum Computation, \\ University of California, Santa Barbara, California 93106, USA
}

(Dated: October 22, 2018)

\begin{abstract}
We investigate the Rabi oscillations decay of a spin decohered by a spin bath whose internal dynamics is caused by dipolar coupling between the bath spins. The decay form and rate as a function of the intra-bath coupling is studied analytically, and confirmed numerically. The decay in general has neither exponential/Gaussian or power-law form, and changes non-monotonically with the intra-bath coupling, decelerating for both slow and fast baths. The form and rate of Rabi oscillations decay can be used to experimentally determine the intra-bath coupling strength for a broad class of solid-state systems.

PACS numbers: 76.30.Da, 03.65.Yz, 76.30.-v, 76.20.+q
\end{abstract}

Measurement of the Rabi oscillations decay is an important step in studying decoherence of quantum systems. For instance, extensive studies of Rabi oscillations in superconducting qubits [1, 2, 3] greatly enhanced understanding of the decoherence caused by the bosonic baths and by the $1 / f$ noise. The most promising directions for improvement were identified, and the decoherence time for superconducting qubits have been extended to microseconds range [4], placing them among most promising solid-state qubit candidates. Recently, much progress has been achieved in experimental implementation of long-living coherent Rabi oscillations in various spin systems: magnetic molecules [5], NV impurity centers in diamond $[\underline{6}, 7,[8]$, rare-earth ions in nonmagnetic host [9], quantum dots [10, 11, 12], to mention a few. Many of these systems are very attractive for basic studies of quantum spin coherence effects, and also constitute promising candidates for quantum information processing, coherent spintronics, or high-precision magnetometry devices, provided that detailed understanding of the decoherence processes will be achieved.

A major decoherence source in these spin systems is the coupling of the central spin (e.g. the electron spin of the NV center in diamond) to other spins present in the sample (environmental bath spins, e.g. the spins of nitrogen atoms in diamond). Moreover, for many relevant spin systems, the direct coupling between the environmental spins is essential, producing internal dynamics of the bath. Here we explore theoretically the influence of such a dynamical spin bath on the Rabi oscillations of the central spin. Avoiding the commonly used framework of generalized Bloch equations [13, 14, 15, 16], we are able to investigate the form and rate of decay as a function of the intra-bath coupling strength. We find interesting behavior of the Rabi oscillations, which contradicts the expectations based on standard Redfield-type analysis: e.g., the slow bath leads to pronounced decay, while for the fast bath the decay rate vanishes but the Rabi frequency becomes renormalized. We demonstrate how the form and rate of the Rabi oscillations decay can be used in experiment to characterize the intra-bath dynamics, and provide a rather simple recipe for analysis of experimental data.

Specifically, we consider the situation of dilute dipolarcoupled bath: in a non-magnetic host crystal, a single central spin of species $S$ (e.g. belonging to a paramagnetic impurity) is coupled to a bath of dilute spins of species $I$ (e.g. belonging to other kind of paramagnetic impurities or to nuclear spins). The coupling between the bath spins is non-negligible, and is caused by dipolar interactions. This situation encompasses a wide range of interesting solid-state spin systems, from Er ions in $\mathrm{CaWO}_{4}$ studied in 1960s [17] to the NV centers in diamond [6, 7, 8] which gained much attention very recently. Moreover, experimental progress now makes possible detailed studies of Rabi oscillations of an individual $S$ spin [6, 8, 18]. We assume that a large magnetic field $B_{z}$ is applied along the $z$-axis (as in standard NMR/ESR settings), leading to Zeeman splittings $\omega_{S}=\gamma_{S} B_{z}$ for the central spin $S$ and $\omega_{I}=\gamma_{I} B_{z}$ for the bath spins $I_{k}\left(\gamma_{S}\right.$ and $\gamma_{I}$ are the gyromagnetic ratios of the $S$ and $I$ spins, respectively). Also, strong Rabi driving field $H_{R}$ is applied at the frequency $\omega_{S}$. The difference $\left|\omega_{S}-\omega_{I}\right|$ is much larger than any other energy scale, and after transformation into rotating frame we obtain a standard secular Hamiltonian for two dipolar-coupled spin species [13, 14]:

$$
H=h_{x} S_{x}+\sum_{k} A_{k} S_{z} I_{k}^{z}+\sum_{k, l} C_{k l}\left(3 I_{k}^{z} I_{l}^{z}-\mathbf{I}_{k} \mathbf{I}_{l}\right)
$$

where $S^{x, y, z}$ and $I_{k}^{x, y, z}$ are the spin operators in the ro- 
tating frame, and $h_{x}=H_{R} / 2$ is the rotating-frame Rabi driving field. The coupling constants $A_{k}=\gamma_{S} \gamma_{I}[1-$ $\left.3\left(n_{k}^{z}\right)^{2}\right] / r_{k}^{3}$ are determined by the positions $\mathbf{r}_{k}$ of the bath spins $I_{k}(k=1, \ldots N)$, where $r_{k}=\left|\mathbf{r}_{k}\right|$ and $\mathbf{n}_{k}=\mathbf{r}_{k} / r_{k}$ (the origin of the coordinate frame coincides with the central spin). Similarly, the intra-bath couplings $C_{k l}=\gamma_{I}^{2}\left[1-3\left(n_{k l}^{z}\right)^{2}\right] / r_{k l}^{3}$ are determined by the vectors $\mathbf{r}_{k l}=\mathbf{r}_{k}-\mathbf{r}_{l}$. Note that the same Hamiltonian (11) can be obtained without external field $B_{z}$ if the transition frequencies $\omega_{S}$ and $\omega_{I}$ are determined by the zero-field splitting (e.g. due to anisotropic interactions). Similarly, the assumption (used below) that $S$ and $I_{k}$ are spins $1 / 2$ is not essential: for larger spins, we can consider each pair of levels as a pseudo-spin 1/2 [13, 14].

Initially the central spin is in the state "up", and the bath is in a maximally mixed state (unpolarized bath at high temperatures), i.e. the initial density matrix of the whole system is $\rho(0)=2^{-N}|\uparrow\rangle\langle\uparrow| \otimes$ $\mathbf{1}_{B}$, where $\mathbf{1}_{B}$ is the $2^{N} \times 2^{N}$ identity matrix. This is appropriate for most experiments (for nuclear spins at temperatures above few $\mathrm{nK}$, for electron spins above tens of $\mathrm{K}$ ). We calculate the time-dependent $z$ component of the central spin $\left\langle S_{z}(t)\right\rangle=\operatorname{Tr} \rho(t) S_{z}$, where $\rho(t)=\exp (-i H t) \rho(0) \exp (i H t)$. In order to see wellpronounced long-living Rabi oscillations, the driving field should be large, so we assume that $h_{x}$ is much larger than all other energy scales. We calculate the oscillations damping in the lowest order in $1 / h_{x}$, treating the second (spin-bath coupling) and the third (bath internal Hamiltonian) terms in Eq. 1 perturbatively, and excluding the bath internal Hamiltonian $H_{B}=\sum_{k, l} B_{k l}\left(3 I_{k}^{z} I_{l}^{z}-\mathbf{I}_{k} \mathbf{I}_{l}\right)$ by the interaction representation transformation. The resulting second-order Hamiltonian

$$
H_{0}=h_{x} S_{x}+S_{x} \hat{B}^{2}(t) /\left(2 h_{x}\right)
$$

where $\hat{B}(t)=\exp \left(i H_{B} t\right) \hat{B} \exp \left(-i H_{B} t\right)$, and the operator $\hat{B}=\sum_{k} A_{k} I_{k}^{z}$.

The evolution of $\hat{B}(t)$ is complex, involving intricate correlations between bath spins. However, exact dynamics of every single bath spin is not important, since $\left\langle S_{z}(t)\right\rangle$ involves tracing over all bath spins. This is typical for many spin-bath decoherence problems, from magnetic resonance to quantum information processing, and many approaches have been developed from the early days of NMR/ESR theory 19, 20] till very recently [21, 22, 23, 24, 25, 26, 27, 28]. All approaches involve a trade-off between quantitative rigour and qualitative understanding. Below, following the works [19, 20], we approximate the effect of the bath by a random field $B(t)$, which is modeled as an Ornstein-Uhlenbek process with the correlation function $\langle B(t) B(0)\rangle=b^{2} \exp (-R t)$, where the dispersion $b=\sqrt{\sum_{k} A_{k}^{2}}$, while the correlation decay rate $R$ is determined by $H_{B}$. This model may be oversimplified for complicated situations, e.g. the description of advanced control protocols requires more sophisticated treatment [24, 28]. However, our direct numeri- cal simulations evidence that this model is quantitatively adequate for description of the Rabi oscillations decay, while providing a clear description of the physics underlying the Rabi oscillations decay, and allowing access to the regimes outside of the Bloch equations framework.

The Hamiltonian (2) reflects a simple physical picture. The zero-order eigenstates of the Hamiltonian (1) correspond to the central spin states $|+\rangle=1 / \sqrt{2}[|\uparrow\rangle+|\downarrow\rangle]$ and $|-\rangle=1 / \sqrt{2}[|\uparrow\rangle-|\downarrow\rangle]$, separated by a large Rabi frequency $h_{x}$. Since $h_{x} \gg b$ and $h_{x} \gg R$, the field $B(t)$ has no spectral components of noticeable magnitude at the Rabi frequency, and does not lead to transition between the states $|+\rangle$ and $|-\rangle$. The only relevant process is the pure dephasing, when the field $B$ destroys the initial phase relation between the states $|+\rangle$ and $|-\rangle$, leading to decay of $\left\langle S_{z}(t)\right\rangle$, so that

$$
\left\langle S_{z}(t)\right\rangle=1 / 2\langle\cos \Phi\rangle=1 / 2 \operatorname{Re}\langle\exp (i \Phi)\rangle
$$

where $\langle\cdot\rangle$ denotes average over all possible realizations of $B(t)$, and the phase

$$
\Phi=h_{x} t+\frac{1}{2 h_{x}} \int_{0}^{t} B^{2}(s) d s=h_{x} t+\Theta
$$

is the total phase difference between the states $|+\rangle$ and $|-\rangle$ accumulated during time $t$, cf. the Hamiltonian (2). The key quantity $M(t)=\langle\exp (i \Theta)\rangle$ is an analytically computable Gaussian path integral over the OrnsteinUhlenbeck process, which gives the answer

$$
\begin{aligned}
\left\langle S_{z}(t)\right\rangle & =1 / 2 \operatorname{Re}\left[M(t) \exp \left(i h_{x} t\right)\right] \\
{[M(t)]^{-2} } & =\exp (-R t)[\cosh P t+(R / P) \sinh P t] \\
& -i \frac{b^{2}}{h_{x} P} \exp (-R t) \sinh P t
\end{aligned}
$$

where $P=\sqrt{R^{2}-2 i b^{2} R / h_{x}}$.

Analysis of Rabi oscillations is often based on Blochtype equations [13] or its generalizations 15], derived for various systems from quantum optics [16] to solid state 15]. It is based on the Redfield-type approach [13, 14], taking into account only the terms which are secular with respect to the Rabi driving $h_{x} S_{x}$. In addition to dephasing, these terms describe the actual transitions between the states $|+\rangle$ and $|-\rangle$, which lead to a longitudinal relaxation (along the $x$-axis) of the central spin with the rate $\Gamma_{l} \sim b^{2} R / h_{x}^{2}$. This rate is of second order in $1 / h_{x}$, and is determined by the spectral density of $B(t)$ at the Rabi frequency $h_{x}$. Also, the generalized Bloch equations, having constant coefficients, always predict the decay to have a (multi)exponential form. In contrast, our results are not limited to the terms secular with respect to Rabi driving, and give the decay rate of the first order in $1 / h_{x}$. The solution (5) predicts the decay which has, in general, no simple form (multiexponential, Gaussian, power-law, etc.) The familiar exponential decay occurs only at special values of $b, h_{x}$, and $R$, see below. 
The effect of the bath internal dynamics may be important even for slow baths, with $R \ll b^{2} / h_{x}$. In this limit,

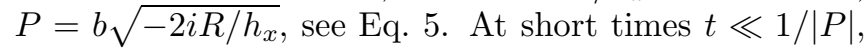
the bath behaves as static, and the Rabi oscillations envelope exhibits non-exponential slow decay of the form $\left[1+\left(b^{2} t / h_{x}\right)^{2}\right]^{-1 / 4}$, in accordance with the exact results obtained earlier [10, 25, 26, 27]. At long times $t \gg 1 /|P|$, the decay has exponential form $\exp \left(-b t \sqrt{R / 4 h_{x}}\right)$, with the rate which decreases very slowly with decreasing $R$. As a result, even for very slow bath the effect of the bath dynamics remains noticeable. In experiments, the above-described behavior of the Rabi oscillations decay can be detected by varying $h_{x}$ (since the ratio of $R$ to $b^{2} / h_{x}$ determines how fast/slow the bath is), and makes it possible to estimate $b$ and $R$.

Another interesting feature of our results is that the decay of Rabi oscillations changes non-monotonously with $R$ : it is fastest for $R \sim b^{2} / h_{x}$, slowing down for both slow and fast baths. This is confirmed by direct numerical simulations, see Fig. 1 and discussion below.

The regime of fast bath, with $R \gg b^{2} / h_{x}$, is even more interesting and unexpected. In this case

$$
M(t)=\exp \left[i \frac{b^{2} t}{2 h_{x}}-\frac{b^{4} t}{4 h_{x}^{2} R}\right]
$$

and the Rabi oscillations exhibit exponential decay with the rate $\Gamma_{t}=b^{4} /\left(4 h_{x}^{2} R\right)$, which vanishes quickly for large $R$. However, it does not mean that the effect of the fast bath disappears. The bath still noticeably affects the central spin, shifting its Rabi frequency by $b^{2} / 2 h_{x}$, and only the decaying part of the bath effect vanishes 29,30 . This slowing of the Rabi oscillations decay is confirmed by direct numerical simulations (Fig. 22).

On the other hand, for fast baths, the transitions between the states $|+\rangle$ and $|-\rangle$ become important. While the dephasing between these states just shifts the Rabi frequency, the transitions lead to longitudinal relaxation along the $x$-axis with the rate $\Gamma_{l} \sim b^{2} R / h_{x}^{2}$. This implies [13] exponential decay of Rabi oscillations with the rate $\Gamma_{l} / 2$, which is comparable with the decay rate $\Gamma_{t}$ caused by the pure dephasing. Above, for simplicity, we omitted the longitudinal relaxation (since it is of order $\left.1 / h_{x}^{2}\right)$, but we can include it using the Redfield-type approach: the answer (5) for $S_{z}(t)$ just has to be multiplied by $\exp \left(-\Gamma_{l} t / 2\right)$. This explains how the system enters the generalized Bloch equation regime at $R \gg b$ : the dephasing effect becomes negligible and the longitudinal relaxation becomes dominant. In experiment, this regime can be identified by comparing $\Gamma_{l}$ and $\Gamma_{t}$ : they would be of the same order, changing as $1 / h_{x}^{2}$ for all sufficiently large $h_{x}$.

To ensure that the physical picture above is adequate for real dipolar-coupled bath, we performed direct numerical simulations of the Rabi oscillations decay. We place the central spin and $N$ bath spins randomly, with uniform
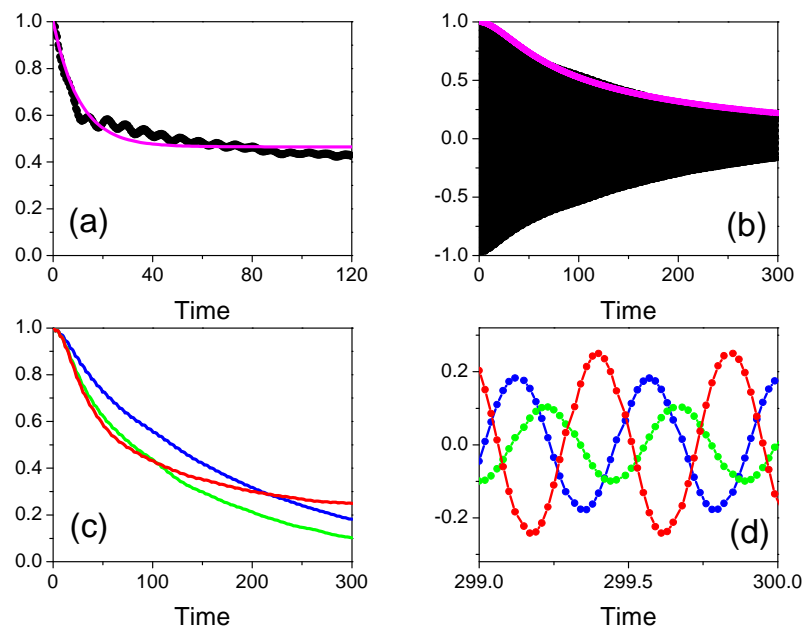

FIG. 1: (Color online). Exact numerical simulations for $N=15$ bath spins. (a) - Correlation function $\langle B(0) B(t)\rangle$ (normalized to 1 at $t=0$ ) obtained from direct simulations (black), see also 32]. Its fitting (green) determines the bath parameters $b_{1}, b_{2}$, and $R$ for each value of the intra-bath coupling scale $E_{B}$ (here $E_{B}=1, b_{1}=0.62, b_{2}=0.58$, $R=0.095$ ). (b) - Rabi oscillations decay for $h_{x}=14.14$, $b=0.85$, and $E_{B}=1$; individual oscillations are not resolved. Numerical results (black) agree well with analytics (magenta line, only the envelope shown). (c) - Envelopes of simulated Rabi decay for $E_{B}=1$ (blue), $E_{B}=0.1$ (green), and $E_{B}=0$ (static bath, red); corresponding individual oscillations near $t=300$ are shown on panel $(\mathrm{d})$ by same colors. Analytical results practically coincide with simulations, and are not shown. The decay rate changes nonmonotonically with $E_{B}$ : the decay is slower for $E_{B}=1$ (blue), and $E_{B}=0$ (red) in comparison with $E_{B}=0.1$ (green). At $t=300$, on panel (d), the oscillation amplitude for $E_{B}=0.1$ (green) is twice smaller than that for $E_{B}=0$.

density $n=1$, inside a cube with the side $(N+1)^{1 / 3}$, central spin being in the center of the cube. All interaction coefficients are calculated according to Eq. 1, using the actual coordinates of the spins. The value $E_{S B}=\gamma_{S} \gamma_{I}$, which determines the strength of coupling between the central spin and the bath, is set to 1, thus defining the energy and time scales for all quantities below. The value $E_{B}=\gamma_{I}^{2}$, which governs the energy scale of intra-bath couplings, is varied, making the bath slower or faster. We simulate the dynamics of the system using two numerical approaches. For small $N$ (e.g., $N=15$ in Fig. 1) we exactly solve of the Schrödinger equation with the Hamiltonian (11) via Chebyshev polynomial expansion [31]. The dipolar-coupled systems with $N>15$ are difficult to model this way (due to exponentially increasing resources requirements), so we use the P-representation sampling 31] for modeling larger baths $(N=159$ in Fig. 2). To compare numerical solutions with the analytics, we calculate the total coupling to a bath $b=\left[\sum_{k=1}^{N} A_{k}^{2}\right]^{1 / 2}$ directly from the positions of the spins (see Eq. 1 and below). The correlation decay rate $R$ requires a separate 

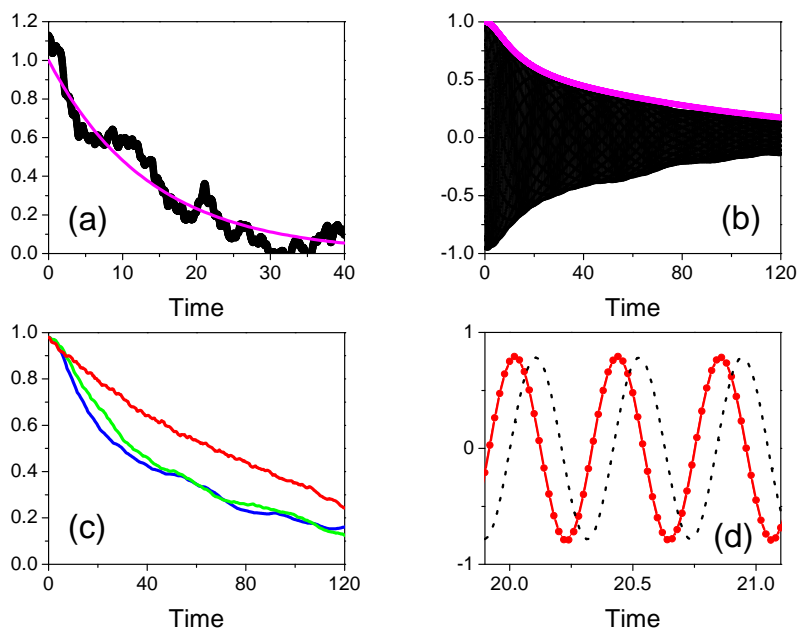

FIG. 2: (Color online). Numerical simulations for $N=159$ bath spins. (a) - Correlation function $\langle B(0) B(t)\rangle$ (normalized to 1 at $t=0$ ) obtained from P-representation simulations (black); its fitting (green) determines the parameter $R$ for each value of the intra-bath coupling scale $E_{B}$ (here $E_{B}=1$ and $\left.R=0.097\right)$. In spite of rather large statistical fluctuations, the parameter $R$ is determined precisely enough to be used in Eq. 5. (b) - Rabi oscillations decay for $h_{x}=15.0, b=1.39$, and $E_{B}=0.1$; individual oscillations are not resolved. Numerical results (black) agree well with analytics (magenta line, only the envelope shown). (c) - Envelopes of simulated Rabi decay for $E_{B}=0.1$ (blue), $E_{B}=1$ (green), and $E_{B}=10$ (red); the longitudinal decay for $E_{B}=10$ is taken into account. Analytical results practically coincide with simulations, and are not shown. The decay rate decreases for faster baths: the decay is slowest for $E_{B}=10$ (red). Individual oscillations for $E_{B}=10$ near $t=20$ are shown on panel (d) in red: their frequency is shifted by $b^{2} / 2 h_{x}=0.064$ from the value $h_{x}=15.0$ (oscillations with frequency $h_{x}=15.0$ are shown by dotted black line to demonstrate the phase difference accumulated since $t=0$ ).

simulation: we calculate $\langle B(0) B(t)\rangle$ and find $R$ by fitting it to a decaying exponent, see also 32. Varying the system parameters in a wide range, we simulated baths with $N=15,59$, and 159 spins, and found good agreement between numerics and analytics; typical results are given in Figs. 112,

The resulting experimental recipe is rather simple. If the decay has a power-law form at shorter times, changing to exponent later, with the decay constants changing as $1 / h_{x}$ and the duration of the two regimes varying with $h_{x}$, then the bath is slow. If the Rabi oscillations decay is exponential, with the rate changing as $1 / h_{x}^{2}$ and the frequency shift varying as $1 / h_{x}$, then the bath is fast. The fast bath can be made slow by strong increase in $h_{x}$.

Summarizing, we studied the decay of the Rabi oscillations of the central spin interacting with a dipolarcoupled dynamical spin bath. Approximating the effect of the bath as a random field (Ornstein-Uhlenbeck process), we find analytically the form of the decay. Valid- ity of the approximation is confirmed by direct numerical simulations. The oscillations decay has interesting features, such as non-monotonic variation of the decay rate with increasing the intra-bath coupling, and slowing down of the decay for fast baths. Studying the behavior of the Rabi oscillations may help in experimental characterization of the dynamical spin bath, and is well within experimental reach e.g. for NV centers in diamond. Work at Ames Laboratory was supported by the Department of Energy - Basic Sciences under Contract No. DE-AC02-07CH11358. We acknowledge support from AFOSR (D.D.A.), FOM and NWO (R.H.). A.E.F. acknowledges support from the Microsoft Corporation.

[1] E. Paladino et al., Phys. Rev. Lett. 88, 228304 (2002)

[2] Y. M. Galperin et al., Phys. Rev. Lett. 96, 097009 (2006)

[3] J. Clarke and F. K. Wilhelm, Nature 453, 1031 (2008)

[4] G. Ithier et al., Phys. Rev. B 72, 134519 (2005); A. A. Houck et al., Phys. Rev. Lett. 101, 080502 (2008).

[5] S. Bertaina et al., Nature 453, 203 (2008)

[6] T. Gaebel et al., Nature Physics 2, 408 (2006).

[7] L. Childress et al., Science 314, 281 (2006).

[8] R. Hanson et al., Science 320, 352 (2008).

[9] S. Bertaina et al., Nature Nano. 2, 39 (2007)

[10] F. H. L. Koppens et al., Phys. Rev. Lett. 99, 106803 (2007)

[11] J. R. Petta et al., Science 309, 2180 (2005)

[12] R. Hanson et al., Rev. Mod. Phys. 79, 1217 (2007).

[13] C. P. Slichter, Principles of Magnetic Resonance (Springer, Berlin, New York, 1990).

[14] A. Abragam, Principles of Nuclear Magnetism (Oxford University Press, Oxford, New York, 1961).

[15] E. Geva et al., J. Chem. Phys. 102, 8541 (1995).

[16] C. Cohen-Tannoudji and S. Reynaud, J. Phys. B 10, 365 (1977).

[17] W. B. Mims, Phys. Rev. 168, 370 (1968)

[18] H. J. Mamin et al., Phys. Rev. Lett. 91, 207604 (2003).

[19] P. W. Anderson and P. R. Weiss, Rev. Mod. Phys. 25, 269 (1953); J. R. Klauder and P. W. Anderson, Phys. Rev. 125, 912 (1962).

[20] R. Kubo, J. Phys. Soc. Japan 17, 1100 (1962); R. Kubo, M. Toda, and N. Hashitsume, Statistical Physics II (Springer, Berlin, New York, 1998).

[21] A. Garg, Phys. Rev. Lett. 74, 1458 (1995).

[22] V. V. Dobrovitski et al., Phys. Rev. Lett. 90, 210401 (2003).

[23] W. A. Coish and D. Loss, Phys. Rev. B 72, 125337 (2005).

[24] R. de Sousa and S. Das Sarma, Phys. Rev. B 68, 115322 (2003); W. M. Witzel and S. Das Sarma, Phys. Rev. B 74, 035322 (2006).

[25] V. V. Dobrovitski et al., quant-ph/0112053 V. V. Dobrovitski et al, HAIT J. Sci. Eng. 1, 586 (2005).

[26] F. M. Cucchietti et al., Phys. Rev. A 72, 052113 (2005).

[27] J. M. Taylor and M. D. Lukin, Quant. Info. Proc. 5, 503 (2006).

[28] W. Yao et al., Phys. Rev. Lett. 98, 077602 (2007); S. 
Saikin et al., Phys. Rev. B 75, 125314 (2007).

[29] This is in clear contrast with the motional narrowing phenomenon, when the free oscillations decay rate vanishes for fast bath [13, 14, 19, 20]. In the motional narrowing case, extremely fast bath does not affect the central spin at all.

[30] This result can be obtained rather universally, for any non-pathological bath, starting from Eq. 2 and assuming that the correlations $\langle B(0) B(t)\rangle$ decay to zero with a rate much faster than $b^{2} / h_{x}$.

[31] W. Zhang et al., J. Phys. Cond. Matter 19, 083202
(2007). P-representation sampling, although not exact, is expected to give accurate results for our problem at large $N$.

[32] For $N=15$, due to finite- $N$ effects, the correlations decay to a finite value, so that the field $B(t)$, in addition to the Ornstein-Uhlenbeck component, has a static component (of order of $1 / \sqrt{N}$ ), and the correlation function has the form $\langle B(0) B(t)\rangle=b_{1}^{2} \exp (-R t)+b_{2}^{2}$, with restriction $b_{1}^{2}+b_{2}^{2}=b^{2}$. Simulations still agree with analytics very well. 